Alternative Francophone vol.1, 10(2016): 8-22

http://ejournals.library.ualberta.ca/index.php/af

\title{
BD, manga, manfra, Nouvelle Manga: distinctions au moyen des matériaux de l'énonciation
}

\author{
Sylvain Rheault \\ Université de Regina
}

\section{Introduction}

Parmi les premiers mangas traduits en France, on compte Gen d'Hiroshima, publié en 1983 par les Humanö̈des Associés puis Akira, publié en 1990 par les Éditions Glénat, qui, cette fois, marque le véritable début de l'entrée massive des mangas sur le marché français. 25 ans plus tard, selon Rocchia, non seulement les mangas traduits représentent environ $40 \%$ des nouveautés (mais seulement $32 \%$ des ventes en volume pour $21 \%$ des recettes), mais il y a aussi de plus en plus de bandes dessinées produites en France qui imitent le style des mangas.

En effet, la popularité du manga a suscité l'émergence de nouveaux artistes talentueux qui imitent le style des mangakas japonais non seulement en France, mais dans le monde entier. Déjà, de solides études sur le sujet ont été publiées. Jacqueline Berndt, en 2010 et 2012, éditait deux volumes de Global Manga Studies. Casey Brienza vient d'éditer récemment, en 2015, Global Manga, un récent recueil d'articles qui enquête justement sur le phénomène mondial de la prolifération dans toutes les cultures des bandes dessinées de style manga produites par des non-Japonais. On donne à ces productions inédites des noms comme OEL [Original English language] manga dans le monde anglophone et «manfra » dans la francophonie, mot valise combinant les termes « manga » et «français ». On peut donner comme exemple de manfras Lanfeust Quest, Actor's Studio.

La découverte des mangas a aussi inspiré le bédéiste Frédéric Boilet à écrire le Manifeste de la Nouvelle Manga, un projet artistique hybride combinant des éléments culturels choisis dans la bande dessinée et le cinéma français ainsi que dans le manga japonais. Le manifeste reflète les opinions de Boilet concernant la BD franco-belge et les mangas japonais. De même, les choix proposés par Boilet pour son projet reflètent une esthétique bien particulière, une hybridité mûrement réfléchie. Afin de «changer la perception du mot», Boilet utilise l'article masculin pour désigner «le manga », soit une production à visée commerciale, et l'article féminin pour «la manga», soit "une $\mathrm{BD}$ japonaise d'auteur, adulte et universelle, parlant des hommes et des femmes, de 
leur quotidien [...]. »

Le projet de Boilet se distinguerait du phénomène du manfra sur de nombreux points, tout comme le manfra se distingue du manga et de la bande dessinée franco-belge. Afin de mettre en lumière les différences, parfois subtiles, entre ces quatre approches artistiques, je propose de les analyser de manière concomitante en utilisant la grille des matériaux qu'on trouve dans $L a$ Clé des procédés. Celle-ci s'organise au moyen de concepts élémentaires limités en nombre, originellement inspirés par la Rhétorique générale du Groupe $\mathrm{Mu}$ qui distinguait les matériaux des opérations. La Clé ordonne de façon structurée toutes les figures de style connues ainsi que de nombreuses notions littéraires de la langue française. La grille permet de distinguer les composantes d'un texte comme les chimistes distinguent les atomes d'une molécule et c'est cette capacité à «disséquer» qui sera exploitée afin de repérer dans chacune des quatre approches artistiques les variations parfois subtiles qui permettront d'opérer des distinctions pertinentes. Je me servirai en particulier de l'une des structures classificatrices de l'ouvrage: les matériaux de l'énonciation. Avec l'énonciation, on passe dans le domaine des aspects du signifié, dans ce qui ne concerne pas directement le contenu, mais tout ce qui peut en modifier la signification. Les procédés de sens qui relèvent de l'énonciation prennent place dans le texte ou dans son environnement. Les matériaux de l'énonciation (locuteur, destinataire, situation, façonnement esthétique, visée, contact), adaptés des notions originellement proposées par Jakobson, permettront d'apporter un éclairage original sur les constituants de la bande dessinée franco-belge, du manga, du manfra et de la nouvelle manga. Cette approche permettra d'arriver, je le souhaite, à une meilleure compréhension des phénomènes d'hybridation, comme celui du manfra.

Dans le présent article, le terme «bande dessinée » (ou BD) désignera les œuvres de la culture franco-belge tandis que «manga» désignera celles produites par la culture japonaise. Le néologisme «manfra» renverra aux œuvres d'inspiration japonaise, mais d'origine francophone tandis que les termes «nouvelle manga » ou «Manifeste », avec une majuscule, référeront au projet de Frédéric Boilet.

\section{Situation}

Le premier matériau que nous allons explorer figure dans les fonctions du langage originellement proposées par Jakobson comme étant la fonction référentielle ou représentative. Il s'agit de la « situation » qui touche le contexte de la communication, la réalité extralinguistique, les circonstances, l'isotopie, 
les espaces mentaux. Il s'agit donc du référent, qui englobe le signifié conceptuel et donne un contexte réel à la communication. Les variations sur la situation, considérée du point de vue de la réalité, donnent par exemple le fantastique, la fantaisie-fiction et la science-fiction. Si le «sens » de la phrase est l'idée qu'elle exprime, la « référence » de la phrase est l'état de choses qui la provoque, la situation de discours ou de fait à laquelle elle se rapporte. La phrase est donc chaque fois un événement différent; elle n'existe que dans l'instant où elle est proférée et s'efface aussitôt. Ainsi, lire une nouvelle dans un journal deux cents ans après les événements rapportés prend un sens tout autre en ce qui a trait à la situation.

Quand on considère la situation, on observe que la bande dessinée, produite dans un pays francophone, propose des référents que la majorité des lecteurs francophones devraient facilement pouvoir décoder, mais que les autres ne comprendront pas sans faire quelques recherches. Par exemple, les Français reconnaîtront une caricature de Jacques Chirac en l'économiste Saugrenus dans l'album Obélix et compagnie. De même, le manga, produit dans l'archipel nippon, propose des référents qui demandent parfois une connaissance approfondie de la culture japonaise. Cependant, Boilet remarque que les mangas ont été abondamment traduits pour le marché francophone et il semble que les référents culturels nippons ne constituent plus, ou pas autant, un obstacle au lectorat non-japonais. Etonnamment, Boilet constate que bien peu de BD franco-belges sont traduites en japonais, ce qu'il attribue non pas à la difficulté de saisir les référents contextuels, mais au fait que la BD mette l'emphase sur les dessins, au détriment de l'histoire. «La BD franco-belge [...] jusqu'aux années 90 se contentait de ressasser les mêmes univers de SF, historiques ou d'aventure [...]» («Manifeste»). Pour Boilet, la différence essentielle réside dans ce que : «à l'opposé de la BD franco-belge, [...] la manga a toujours privilégié le quotidien» («Manifeste»). Mais qu'est que le « quotidien » pour Boilet? "Quand elle parle de quotidien, la BD devient non seulement plus universelle (l'universalité est le plus souvent dans sa cuisine ou au fond du jardin, et beaucoup plus occasionnellement sur Mars ou Alpha du Centaure), elle devient aussi, aux yeux des lecteurs étrangers, plus "française". » Il faut avouer que la définition du " quotidien » reste assez vague, mais quand on prend en compte l'ensemble du Manifeste, on comprend que le «quotidien» s'oppose à l'aventure et que les ingrédients n'en sont pas les événements uniques, les aventures extraordinaires ou des individus dotés de qualités supérieures, mais plutôt les interactions usuelles entre des humains que l'on pourrait rencontrer dans la rue. D'autre part, les œuvres citées par Boilet comme exemples de nouvelle manga (Yoshiharu Tsuge, Vanyda), en y incluant les siennes, suggèrent que le «quotidien » concerne plus particulièrement les 
relations adultes. En effet, sans ce critère «adulte», on pourrait avancer qu'il existe une certaine forme de "quotidien » chez Astérix, puisqu'un album sur deux se passe dans l'intimité du village des Gaulois. On y voit les villageois interagir, on y voit Astérix manger chez lui, dans sa salle à manger, mais on n'est pas témoin de situations adultes.

Quant au manfra, au point de vue de la situation, on peut en dire qu'il est produit dans la francophonie, tout comme la $\mathrm{BD}$, mais qu'il puise aussi dans la culture japonaise et notamment dans la culture graphique, celle des codes iconiques. On peut donc s'attendre à ce que les référents soient à portée d'un lectorat francophone amateur de mangas. Nous reviendrons sur cette question plus loin

\section{Locuteur}

Jakobson appelle «émetteur» la source du message au centre de la fonction expressive, qui se manifeste dans les épanchements de sentiments et les opinions exprimées. Suivant l'usage de la Clé des procédés, nous l'appellerons «locuteur». C'est l'entité qui peut dire « je ». Il faut le prendre non pas comme existence, en temps qu'être charnel, mais comme concept, comme une manifestation dans l'œuvre. La présence du locuteur dans le $9 \mathrm{e}$ art peut se détecter par une manière toute personnelle de traiter le matériau artistique, par un style particulier, parfois simplement par une signature.

Quelle trace du locuteur peut-on trouver dans les bandes dessinées de l'Europe francophone? Avant les années 1970, on reconnaissait à sa couverture un album des Schtroumpfs et on faisait peu de cas de Peyo, l'auteur. Depuis, on a donné à certains et certaines bédéistes le statut de vedette. Pensons à Claire Brétécher ou à Gotlib. Boilet constate qu'une «nouvelle BD » est née en France, en réaction à la $\mathrm{BD}$ «SF/héros/action» (Manifeste), en particulier les publications de maisons de publication moins commerciales comme l'Association ou Ego comme X. Boilet emploiera le terme de «BD d'auteur» dans son Manifeste. Désormais, on vend certaines œuvres en mettant en évidence sur la couverture le nom du ou de la bédéiste et une œuvre reste indissociable de son instance créatrice. Ainsi, on met sur un pied d'égalité l'auteure Marjane Satrapi et sa création: Persépolis. D'autre part, le caractère autobiographique d'une bande dessinée, donc la présence même de l'auteur dans son récit, constitue depuis quelques années un atout. À ce propos, Bart Beaty observe dans son livre Unpopular Culture, que, au sein des $\mathrm{BD}$ indépendantes, l'autobiographie (incluant la fiction autobiographique) est devenue un genre dominant. Beaty cite surtout des bédéistes publiés par la 
maison d'édition L'Association, mais on peut aussi penser à des auteurs comme Guy Delisle et Pénélope Bagieu. On apprécie le caractère authentique de ces productions. Boilet reconnaît la BD d'auteur entre autres par la prédominance «des histoires souvent basées sur le quotidien, autobiographique ou imaginaire» («Manifeste»). Enfin, il faut ajouter que le ou la bédéiste francophone a la nationalité belge ou française.

Lorsqu'il s'agit du manga japonais, la fonction du locuteur n'est pas si simple. En effet, il faut tenir compte de la relation professionnelle incontournable entre l'auteur et le chargé d'édition, établie dans les années 1980 (Bouissou 110). Le chargé d'édition, dont le rôle a aussi été décrit par Sharon Kinsella (8-9; 50), s'assure que l'auteur respecte les échéances et lui apporte le soutien nécessaire, et cela va parfois même jusqu'à lui donner un coup de main pour boucler ses planches avant l'échéance. L'impact du chargé d'édition sur l'œuvre est immense et il faut en tenir compte. Son nom figure d'ailleurs dans les pages éditoriales des mangas. Et puis, bien sûr, le ou la mangaka, de même que le chargé d'édition possèdent la nationalité japonaise.

Quant aux mangas traduits, la plupart sont choisis en fonction de leur popularité au Japon ou parce qu'une version en dessin animé joue à la télévision. Dans certains cas, cependant, les éditeurs choisiront des mangas en fonction d'un auteur, comme Takahashi Rumiko ou Mizuki Shigeru. Quant aux bandes dessinées francophones traduites en japonais, Boilet rapporte qu'au Japon, si les noms de Mœbius et Bilal sont connus, "pour les rares Japonais qui ont vu leurs œuvres, c'est surtout pour la qualité de leur dessin qu'on les apprécie ». (« Manifeste»).

Boilet affirme, d'entrée de jeu, dans son Manifeste que la nouvelle manga est « une initiative d'auteur (par opposition aux initiatives d'éditeur ou de librairie d'import, débouchant immanquablement sur des traductions — ou des importations - du tout-venant des séries à succès) ». Boilet se fait fort de donner l'exemple, en suivant la voie de l'autobiographie et en nous convoquant non seulement dans le quotidien de son logis, mais jusque dans l'intimité de ses ébats amoureux. Enfin, le ou la bédéiste qui participe au projet de la Nouvelle Manga peut être aussi bien de nationalité japonaise que d'un pays de la francophonie.

Quant au manfra, il s'agit simplement, pour le locuteur (l'auteur), d'être né dans un pays francophone. On ne peut pas créer de manfra si on est né au Japon. La façon de travailler reste aussi européenne, comme celle des auteurs de BD. 
Alternative Francophone vol.1, 10(2016): 8-22

http://ejournals.library.ualberta.ca/index.php/af

\section{Destinataire}

Le destinataire représente la personne morale à qui on peut dire «tu». Jakobson la nomme "fonction injonctive », parce qu'on cherche à provoquer une réaction chez l'interlocuteur, parce qu'on veut obtenir quelque chose de lui. Une œuvre peut avoir pour cible un public bien précis, ce qui aura un impact sur le contenu et même sur le genre. Les amateurs de fantastique rechercheront le fabuleux tandis qu'on proposera aux enfants des contes de fées.

Boilet affirme que «les univers de SF ou d'action des bandes dessinées franco-belge et américaine ne ciblent quasiment que les adolescents masculins» («Manifeste»). Cela implique que la bande dessinée franco-belge néglige deux grands publics potentiels : les lecteurs adultes ainsi que le lectorat féminin. Évidemment, il s'agit d'une généralisation, mais nous la prendrons telle qu'elle se présente pour l'instant. On doit ajouter que le public de la BD franco-belge lit en français et comprend les références à la culture francobelge.

Au Japon, les maisons d'édition de mangas, cherchant à développer davantage le marché, ont, avec le temps, augmenté de plus en plus l'étendue du lectorat. Selon Bouissou (101), il existe aujourd'hui de nombreuses niches, que ce soit pour les filles ou les garçons, qu'ils soient enfants, adolescents ou jeunes adultes, ou encore qu'ils aient une passion pour des activités spécifiques comme le sport, le mah-jong ou la pornographie. Presque tout le monde peut y trouver son compte. Le public original pour lequel le manga est créé, lit le japonais et est au fait des références culturelles propres à l'archipel nippon. D'autre part, au Japon, le public des magazines décide de la survie d'une série et du destin des personnages en participant aux sondages qu'on trouve dans les magazines. La maison d'édition utilise cette information pour terminer une série ou encore étirer une histoire qui fonctionne bien. De même, le chargé d'édition peut accéder à la demande populaire et imposer à un auteur de remettre le personnage d'une série sous le feu des projecteurs. Enfin, il existe au Japon un public de passionnés de mangas qui, en plus de les consommer, discutent entre eux du fil de l'histoire de leurs séries préférées. Il s'agit de la génération otaku que Bouissou décrit comme une culture de «base de données » (130).

Pour ce qui est des mangas traduits, il faut d'abord se rendre compte que le public francophone qui ne lit pas le japonais n'a accès qu'aux œuvres 
choisies par les maisons d'édition qui en font la traduction. On préférera publier d'abord des mangas qui sont portés par une série d'animation, des séries qui sont populaires au Japon, en particulier auprès d'un public adolescent, les plus grands consommateurs, et dont les référents restent assez universels. Mais Boilet considère que «L'essentiel de la manga traduite en France depuis plus de dix ans est une manga commerciale pour adolescents [...] » (« Manifeste »). Il s'agirait donc essentiellement du même public que pour la BD franco-belge. Cependant, il faudrait reconnaître une grande percée : l'apparition du shojo manga a permis la conquête du lectorat adolescent féminin, ce qui a incité des jeunes femmes à se lancer dans la carrière de bédéiste, comme Vanyda et Aurélia Aurita en France ainsi que Zviane au Québec (Rheault). Cela constitue un phénomène social important. Pour en revenir au public de mangas traduits, il s'agit d'un lectorat qui lit le français et qui est capable d'embrasser les références culturelles japonaises, incluant les codes graphiques, sans nécessairement toutes bien les saisir.

On peut compter, parmi les mangas qui sont extrêmement populaires au Japon et qui ne sont pas (encore) traduits en français, les mangas politiques, les mangas à propos des salarymen ou des O.L. ${ }^{1}$ Une série comme Kachou Shima Kousaku, (Chef de section Shima Kousaka, publié périodiquement entre 1983 et 1992) par Hirokane Kenshi, nous fait entrer dans l'univers des grandes compagnies japonaises, mais ne semble pas avoir trouvé de public en France.

Boilet définit le public de la nouvelle manga comme étant des «lecteurs curieux, ouverts, qui lisent une bande dessinée comme ils liraient un roman ou vont au cinéma » ("Manifeste»). Il faut l'opposer à un public « otaku», c'est-à-dire essentiellement fan de mangas commerciaux. On note que certaines des œuvres de Boilet sont bilingues : japonais et français. Boilet envisage le public de la nouvelle manga non pas en fonction de la langue, mais en fonction de l'ouverture et de la curiosité pour les arts narratifs. Un public intellectualisant en quelque sorte.

Enfin, le public cible du manfra est essentiellement les francophones qui sont déjà des amateurs de mangas. L'un des défis, comme le note François Oulac dans un article publié en ligne, c'est que le manfra apparaît comme "pas assez authentique pour les puristes du manga japonais, trop japonais pour les fans de BD franco-belge ». Néanmoins, le lectorat existe et il lit surtout en français tout en étant familier avec les codes graphiques du manga.

${ }^{1}$ Office Ladies, employées d'une compagnie cantonnées dans des rôles de soutien, en servant le thé, par exemple. 
Alternative Francophone vol.1, 10(2016): 8-22

http://ejournals.library.ualberta.ca/index.php/af

\section{Contact}

Le contact touche ce que Jakobson appelle la fonction phatique et est liée au "canal» de communication. Il s'agit d'établir un contact, comme par exemple l'interpellation ou l'envoi d'un signal S.O.S. à la radio, ou d'employer un canal de communication particulier, comme le livre ou la tablette électronique. Le canal choisi a un impact dramatique sur la forme et le contenu de l'œuvre. En guise d'illustration, une formule comme « je t'aime » ne passera pas de la même façon au téléphone, dans une lettre ou en personne.

Le contact, pour la $\mathrm{BD}$, passe avant tout par l'attrait qu'exerce la page couverture. Cette dernière donne au lecteur potentiel des informations sur le contenu de l'album, sur l'auteur, sur la maison d'édition, au besoin sur la série. La page couverture doit séduire le lectorat en l'incitant à la lecture et en provoquant l'achat. Boilet raconte que la BD franco-belge, dont Moebius et Bilal sont les représentants, n'a connu que peu de succès au Japon, malgré " une publicité orchestrée depuis la fin des années 80 par les éditeurs, la presse, les libraires et les institutions françaises » (« Manifeste »).

Pour distinguer la manière dont s'établit le contact, comparons les couvertures de BD franco-belges avec celles des mangas. Les couvertures de Tintin montrent le héros, certes, mais son corps n'occupe habituellement qu'un petit pourcentage de la surface. Le reste de l'image montre généralement un décor ou des objets qui suggèrent un endroit précis, qu’on peut reconnaitre. L'emphase est mise sur les lieux et sur les voyages. Les couvertures de la série Naruto, quant à elle, présentent presque toujours le héros en gros plan. Le corps de Naruto occupe un pourcentage beaucoup plus important de la surface et il y a presque toujours d'autres personnages avec lui. Tous et toutes sont montrés dans des poses dynamiques et il n'y a habituellement que très peu d'indices concernant le lieu et le moment de l'aventure. L'emphase, dans les mangas, porte de toute évidence sur les personnages héroïques et leurs interactions. Le sondage que nous avons effectué avec l'aide d'un fureteur sur l'Internet n'est pas exhaustif, certes, mais c'est ce que révèlent les quelques centaines de pages couvertures de manga que nous avons pu trouver. L'aspect physique du manga a aussi un rôle à jouer. Le format "tanköbon » plus petit que l'album, présente néanmoins un nombre de pages presque six fois supérieur. Cela suggère une narration beaucoup plus longue. Les mangas traduits suivent généralement la pratique japonaise, incluant le format ainsi que la lecture de droite à gauche.

Les pages couvertures de manfras que l'on peut trouver sur l'Internet 
ne diffèrent pas beaucoup de celles des mangas. Notons cependant que le manfra n'est pas nécessairement offert en format tankōbon. Il peut adopter le format album (Les Légendaires de Patrick Sobral) ou un format intermédiaire, comme Celle que je ne suis pas de Vanyda.

Comme pour le manfra, les pages couvertures des albums de Boilet ou d'auteurs identifiés comme adhérents à la nouvelle manga suivent tantôt la pratique européenne, tantôt l'usage nippon. Il n'y a pas de constantes, mais il y a cependant une différence notable: les personnages n'occupent pas toute la surface de la couverture et on trouve sur celle-ci des indices sur le lieu et le moment, comme c'est le cas pour L'Épinard de Yukiko. Les personnages sont montrés dans des poses relâchées, suggérant l'introspection. L'emphase porte nettement sur le quotidien, c'est-à-dire qu'il n'y sera pas question d'aventure. Encore une fois, il est intéressant de noter que les pratiques retenues pour la nouvelle manga constituent une espèce de collage d'éléments culturels tantôt propres à la $\mathrm{BD}$, tantôt propres au manga.

\section{Façonnement esthétique}

Le façonnement esthétique, ou ce qui relève de la fonction poétique de Jakobson, se définit par un ensemble de choix effectués en regard du travail de l'artiste et qui touchent la « beauté » du message. On associe souvent ces choix à une époque et à une région et cela permet, par exemple, de reconnaître une « école» (classicisme, baroque, romantisme, surréalisme, existentialisme, etc.), un «style » (à la manière de Molière, Proust, Hergé, etc.) ou un "genre » (sonnet, roman, comédie, tragédie, shōnen, shōjō, etc.)

L'opinion de Boilet sur la BD franco-belge est sans équivoque: «Comparée à la manga, la $\mathrm{BD}$ privilégie le dessin. Ses auteurs sont avant tout des illustrateurs, souvent plus préoccupés par le graphisme que par le scénario» (Manifeste). Boilet reproche aussi à la BD franco-belge de se cantonner dans le «cadre strict du 46 pages couleur à suivre» ("Manifeste »). Il n'est pas seul à avoir cette opinion. Bart Beaty, dans Unpopular Comics, écrit que:

The hardcover comic book is so central to the history of European comic book production that for many critics and historians it is virtually impossible to think about that history except as a continuity of album production over time, with a few exceptions. For generations the primary aspiration of European comic book producers has been to create a successful comic book series that can be extended indefinitely in a continuity of forty-six-page volumes. The industrial logic of the largest Franco-Belgian publishing houses - which dominate the European market — has been rooted, as Thierry Groensteen has noted, in the eternal return of popular characters in identifiable and marketable comic book albums. (171) 
Le succès économique des $\mathrm{BD}$ franco-belge consisterait donc à s'en tenir à une recette bien rodée. Les idées innovatrices doivent donc utiliser d'autres voies de publication.

Au contraire, selon Boilet, «la bande dessinée japonaise accorde une importance particulière à l'histoire (ampleur des récits, variété des thèmes) et surtout à la narration (sa fluidité, sa technique pour suggérer les sensations, les sentiments)»(«Manifeste »). Il s'agit même de la toute première phrase du Manifeste. Entre les deux composants de la narration graphique, Boilet préfère manifestement le bon scénario au bon dessin. Il faut cependant noter que, ni pour la BD ni pour le manga, Boilet ne discute du style dans son manifeste puisque pour lui la différence essentielle réside dans les oppositions narration/dessin et quotidien/aventure. Au contraire, Bouissou (147 et sq.), explique longuement les principes graphiques et narratifs du manga, en particulier au miroir des traditions européennes. Notons, entre autres, les moyens de représenter les émotions, la manière de dessiner les corps et les visages ou les codes de mouvement. Ces codes graphiques, développés au Japon au cours des années, sont maintenant offerts au reste du monde.

Du point de vue de l'esthétisme, c'est en grande partie l'adoption des codes graphiques du manga qui définit le manfra. Les créateurs et créatrices font leurs le canon de personnages comme Son Goku de la série Dragonball ou Tohru Honda de la série Fruit Basket. grands yeux, nez presque inexistant, bouche minuscule ou inexistante, les chevelures présentant de grandes variations de couleurs, de volume et de style.

Enfin, pour le projet de la nouvelle manga, Boilet, met complètement de côté l'aspect dessin. Ce qui importe, c'est que l'histoire prime sur le dessin et qu'on mette en scène le quotidien plutôt que l'aventure. Dans cette optique, le style du dessin aussi devient secondaire et devient une marque identifient la créatrice ou le créateur.

\section{Visée argumentative}

La visée argumentative définit le véritable enjeu de la communication. Elle peut être implicite, ce qui permet de jouer sur le sens véritable d'un message, dont le contenu servira de distracteur. Cette fonction, que Jakobson considère comme faisant partie du message, est ici considérée à part. Distincte de l'énoncé, la visée permet de conceptualiser des procédés comme ceux qui 
s'apparentent au mensonge. On peut penser au renard de la célèbre fable qui « dit» (énoncé) au corbeau qu'il a une belle voix, alors qu'en fait il «pense » (visée argumentative) à obtenir le fromage. La visée de toute publicité est de vendre, mais de nombreuses annonces jouent sur cette dichotomie en vantant un style de vie (énoncé) au lieu de demander directement au consommateur d'acheter le produit (visée argumentative). C'est le cas pour presque toutes les annonces de vêtements ou de voitures. La convergence des procédés est une forme de visée qui détermine le travail à faire sur chacun des matériaux. Pour le médium de la bande dessinée, on peut donner à un sujet une importance accrue en lui accordant une surface relative plus grande ou en lui faisant occuper une meilleure place sur la planche.

Expliquant d'où vient le projet de son livre dans Unpopular Comics, Bart Beaty observant le marché de la $\mathrm{BD}$ franco-belge, dégage "[the] tension that exists between comic books conceived as popular culture and comic books conceptualized as art» (3). La distinction est visible dans les tirages que cite Beaty: Un album de Titenf de Philippe Chappuis (dit «Zep») se vend facilement à 370,000 exemplaires tandis qu'on n'imprimera que 150 copies d'une œuvre obscure comme Odette et l'eau de Nadia Raviscioni (4). L'industrie de la $\mathrm{BD}$, en soi, a une visée commerciale : il s'agit de vendre le plus possible d'albums à un public qu'on cherche à fidéliser le plus possible. En parallèle, une $\mathrm{BD}$ non commerciale se développe, adoptant une visée purement artistique, qui propose des expérimentations graphiques et narratives. La visée commerciale devient alors secondaire. Boilet mentionne qu' : «avec des éditeurs comme l'Association ou Ego comme X, une $\mathrm{BD}$ au quotidien est née dans les années 90 , précisément en réaction à cette $\mathrm{BD}$ illustrative et commerciale qui a figé les années $80 »$ («Manifeste »).

Si on parle de commercialisation, l'industrie du manga japonais est la championne incontestable. Les maisons d'édition de manga japonais visent un maximum de ventes et, pour ce faire, s'informent constamment des goûts du public et s'évertuent à conquérir de nouvelles niches, comme les femmes et les adultes. Cependant, tout comme en Europe, il existe aussi des mangas à visée expérimentale plutôt que commerciale, comme ceux qu'on trouve dans la revue Garo (Bouissou 389).

Maintenant, si on considère la nature du manfra, quelle pourrait en être la visée? On pourrait affirmer que la créatrice ou le créateur s'adonnant à ce genre hybride aspire certainement à un maximum de ventes. Il ne s'agit pas ici d'expérimenter ou d'innover, mais d'exploiter des procédés graphiques et narratifs qui ont fait leur preuve, autant au Japon qu'en Europe, par le biais des 
traductions. Encore une fois, il semble que le public ne perçoive pas le manfra de la même façon. La nature hybride du manfra, aux yeux du public francoeuropéen, en fait un genre artificiel, non authentique (Oulac), et souvent expérimental, destiné à un lectorat réduit de fans inconditionnels seuls capables de l'apprécier.

Il en va tout autrement de la visée de la nouvelle manga. Au départ, la nouvelle manga se range derrière une certaine visée artistique puisqu'il s'agit de favoriser certains ingrédients qui ne font pas partie de la recette magique du succès des ventes en France et en Belgique, comme le quotidien et la narration plutôt que l'aventure et le dessin.

\section{Conclusion}

Après cette exploration, qu'est-ce que les matériaux de l'énonciation ont pu nous révéler sur les $\mathrm{BD}$, les mangas, les manfras et la nouvelle manga? Les différences entre la $\mathrm{BD}$ et les mangas ont déjà été abondamment analysées et mises en lumière, et il n'y a rien de ce côté qu'on ne suspectait déjà. Le manfra, malgré son dessein avoué d'adopter sans réserve toutes les stratégies qui ont fait le succès du manga au Japon, garde définitivement de fermes racines en Europe. D'entrée de jeu, l'auteur(e) de manfra et son public sont européens, et la culture qu'ils partagent s'immisce nécessairement dans l'œuvre. Si le style du manfra, autant sur la page couverture qu'à l'intérieur du livre, s'inspire clairement des codes graphiques du manga, il arrive à l'œuvre d'adopter aussi des codes de la BD franco-belge, incluant le format. Bref, le manfra se caractérise d'abord par son hybridité et la perception plutôt réservée du public semble confirmer ce jugement. Cette hybridité, malheureusement, semble entraîner, du moins pour l'instant, une certaine mise à l'écart de la part du grand public. Quant à la nouvelle manga, le projet initial était, justement, une hybridité intentionnelle, obtenue en métissant des éléments choisis de la BD (surtout le dessin) et du manga (surtout la narration). Il fallait, selon la tradition européenne, donner la primauté à l'auteur tout en cherchant à séduire de nouveaux lectorats, comme c'est le cas au Japon. Il fallait donner la primauté à la narration et au quotidien, comme au Japon, dans certains types de mangas, tout en publiant dans le format européen. Il semble que la visée initiale, axée sur une certaine hybridité, soit devenue une visée surtout artistique, comme en fait foi la mise à jour du Manifeste, faite en 2007, où Boilet constate que :

La Nouvelle Manga est aujourd'hui plus largement une initiative d'auteurs qui cherche, en créant des ponts entre les créateurs, les éditeurs et les lecteurs de toutes origines, à promouvoir une bande dessinée universelle, à présenter ce que manga, comics et $\mathrm{BD}$ ont de meilleur et non pas seulement de vendeur, ceci plutôt dans le 
registre universel du quotidien, autobiographique, documentaire ou fictionnel.

Si le manfra semble souffrir de son caractère hybride, la nouvelle manga apparaît déjà comme une réussite artistique. En fait, l'hybridité n'est-elle pas l'essence même de l'invention, comme le suggère Arthur Koestler dans The Act of Creation? Manfra et nouvelle manga constituent tous les deux des expérimentations d'amalgame en soi et pour reprendre les termes de Koestler, la « confrontation » qui résulte de la mise en relation des procédés de la BD et du manga aboutit à une nouvelle expérience esthétique. Manfra et nouvelle manga, chacun à leur façon, constituent donc des esthétismes novateurs en émergence dont il vaut la peine de surveiller l'évolution. 
Alternative Francophone vol.1, 10(2016): 8-22

http://ejournals.library.ualberta.ca/index.php/af

\section{Bibliographie}

Beaty, Bart. UNpopular Culture - Transforming the European Comic Book in the 1990s. University of Toronto Press. 2007.

Berndt, Jacqueline, ed. Comics Worlds \& the World of Comics - Global Manga Studies, vol. 1. Kyoto Seika University. 2010.

Berndt, Jacqueline, ed. Intercultural Crossovers, transcultural Flows: Manga/Comics Global Manga Studies, vol. 2. Kyoto Seika University. 2012.

Blondel, Julien; Collao, Camilo. Actor's Studio. Les Humanoïdes Associés. 2007. Boilet, Frédéric. Manifeste de la Nouvelle Manga. http://www.boilet.net/fr/nouvellemanga.html. Consulté le 30 mars 2016.

- La Nowvelle Manga en 2007. http://www.boilet.net/fr/nouvellemanga.html. Consulté le 30 mars 2016.

Bouissou, Jean Marie. Manga. Histoire et univers de la bande dessinée japonaise. Picquier. 2010.

Brienza, Casey. ed. Global Manga - "Tapanese" Comics without Japan? Ashgate Publishing Limited. 2015.

Canário, Tiago. "On Everyday Life: Frédéric Boilet and the Nouvelle Manga Movement". Global Manga - "Japanese" Comics without Japan? Brienza, Casey. ed. Ashgate Publishing Limited. 2015. pp. 115-132.

Dupriez, Bernard et Rheault, Sylvain. La Clé des procédés littéraires. http://www.cafe.etfra.umontreal.ca/cle/index.html. Consulté le 30 mars 2016.

Goscinny, René; Uderzo, Albert. Obélix et Compagnie. Dargaud. 1976.

Gravett, Paul. Manga: Sixty Years of Japanese Comics. Lawrence King. 2004.

Groensteen, Thierry. L'Univers des Mangas. Casterman. 1991. Système de la bande dessinée. PUF. 1999.

Groupe Mu. Rhétorique générale. Seuil. 1982.

Guibert, Xavier. "Le Manga et son histoire vus de France : entre idées reçues et approximations", Comicalités (2012). Consulté le 7 mars 2016. http://comicalites.revues.org/733.

Hirokane, Kenshi. Kachou Shima Kousaku (Chef de section Shima Kousaku). Koudansha. 1983-1992. 17 volumes.

Ingulsrud, John E; Allen, Kate. Reading Japan Cool - Patterns of Manga Literacy and Discourse. Lexington Books. 2009.

Jakobson, Roman. Essais de linguistique générale. Éditions de minuit. 1963.

Katsuhiro, Otomo. Akira. Koudansha. 1982-1990. 6 volumes.

Kinsella, Sharon. Adult Manga - Culture \& Power in Contemporary Japanese Society. 
University of Hawai'i Press. 2000.

Koestler, Arthur. Le Cri d'Archimède. (The Act of Creation). Calmann-Lévy. 1965 (1964).

Lullabi, Ludo. Lanfeust Quest. Soleil. 2007.

Nakawaza, Keiji. Hadashi no Gen [Barefoot Gen]. Shueisha, 1973-1974.

Oulac, François. «Le Manga français peut-il sortir de sa niche? » in Slate.fr. http://www.slate.fr/story/113867/manga-francais-niche. Consulté le 7 mars 2016.

Rheault, Sylvain. "Les Choix des créatrices de bande dessinée ». Voix Plurielles, vol. 9, no 2, 2012. pp. 91-104.

Rocchia, Simon. "Le marché du manga en France». 1er septembre 2014. http://mondedulivre.hypotheses.org/1929. Consulté le 11 mars 2016.

Schodt, Frederik L. Manga! Manga! The World of Japanese Comics. Tokyo: Kodansha. 1984.

-. Dreamland Japan. Writings on Modern Manga. Berkeley: Stone Bridge Press. 1996.

Wikipedia. Article «Manfra ». https://fr.wikipedia.org/wiki/Manfra. Consulté le 7 mars 2016. 